\title{
Comparison of the Responses of New Reduced- Size Calorimetric Cells Made of Different Structure Materials for High Energy Deposition
}

\author{
A. Volte ${ }^{1}$, C. Reynard-Carette ${ }^{1}$, A. Lyoussi ${ }^{2}$, J. Brun $^{1}$, M. Carette ${ }^{1}$ \\ ${ }^{1}$ Aix Marseille Univ, Université de Toulon, CNRS, IM2NP, Marseille, France \\ ${ }^{2}$ CEA, DEN, DER, Instrumentation Sensors and Dosimetry Laboratory, Cadarache, France \\ christelle.carette@univ-amu.fr
}

\begin{abstract}
This paper concerns experimental and numerical works on a new differential calorimeter called CALORRE and validated recently under irradiation conditions in MARIA reactor at low nuclear absorbed dose rate level. Works focus on a specific configuration of CALORRE which was designed especially for the measurement of high nuclear energy deposition rates inside Material Testing Reactors. Due to the high level, a new calibration system was fabricated in order to determine the response of the new configuration under laboratory conditions for a very wide range of electrical power never applied in the literature. The response of the new configuration can be considered linear for this very wide range of electrical power. An analytical calculation shows the contribution of each heat transfer in specific zones. 3D thermal simulations performed by means of COMSOL Multiphysics under irradiation conditions give the predictions of the calorimeter behavior under real conditions (up to $20 \mathrm{~W} \cdot \mathrm{g}^{-1}$ ).
\end{abstract}

Index Terms-Calorimeter, Nuclear Dose Rate, On-line Measurements, Calibration, Irradiation campaign.

\section{INTRODUCTION}

$\mathrm{R}$ esearch in the nuclear field lacks some experimental data essential for numerical simulations in harsh conditions (including intense neutron and photon fluxes and high displacement per atom per year) and thus to better understand the behavior of inert materials or nuclear fuels under irradiations: understanding important for safety challenges, longevity of existing nuclear power plants (NPPs) and development of new concepts of NPPs. Hence, new very efficient instrumentation and devices are required to measure several crucial parameters on-line with a high quality and accuracy, in particular in the framework of the construction of a new material testing reactor (MTR) in Europe (CEA Cadarache, France), the Jules Horowitz Reactor (JHR). Among these parameters, there is the nuclear absorbed dose rate. It is equal to the energy deposition rate per units of mass induced by radiation interactions with matter. It is a key parameter to predict temperature of material samples inside the entire reactor, to design core or reflector experiments and devices, and finally to interpret in-pile experimental results. The JHR with a nominal power of 100MWth will deliver a high fast neutron flux $\left(5.5101^{4} \mathrm{n} \cdot \mathrm{cm}^{-2} \cdot \mathrm{s}^{-1}\right.$ from an energy of $1 \mathrm{MeV}$ ) leading to a high accelerated ageing (up to $16 \mathrm{dpa} /$ year) and a high nuclear absorbed dose rate (up to $20 \mathrm{~W} \cdot \mathrm{g}^{-1}$ ). This new latter value, not reached in existing European MTRs, implies improvements and innovations of dedicated sensors used to direct measurements such as in-pile calorimeters. Nowadays, two distinct sensors are used for the nuclear absorbed dose rate measurement in MTRs [1-5]: differential calorimeters and single-cell calorimeters including gamma thermometers. In these two cases, the nuclear absorbed dose rate is quantified inside a dedicated reactor channel thanks to temperature measurements (absolute or differential temperatures) and the use of calibration curves achieved preliminary, under laboratory conditions (out-of-reactor) from steady thermal states when heating elements are integrated inside the calorimeter or from non-stationary thermal states for a single-cell calorimeter without heater. At present, one crucial objective corresponds to the measurement of the nuclear absorbed dose rate up to $20 \mathrm{~W} \cdot \mathrm{g}^{-1}$ since no current calorimeter have been designed for such value (up to $15 \mathrm{~W} . \mathrm{g}^{-1}$ for gamma thermometers and $13 \mathrm{~W} \cdot \mathrm{g}^{-1}$ for differential calorimeters). Consequently, Aix-Marseille University and CEA into the framework of a joint laboratory called LIMMEX are involved in research works on instrumentation and measurement methods for online quantification of nuclear and thermal parameters in Material Testing Reactors (MTRs). In particular, studies focus on calorimeters (single-cell and differential calorimeters) used for the quantification of the nuclear absorbed dose rate [2-7]. Research works coupling experiments (under laboratory and real conditions) with thermal numerical works (1D calculations and 3D simulations) allow the design, the characterization, the calibration, the qualification and the miniaturization of various calorimeters owning different metrological advantages (sensitivity, range, size...). This approach led to the design of a new compact calorimetric cell called CALORRE characterized under laboratory conditions and to a first prototype validated under irradiations condition in the MARIA reactor at low nuclear absorbed dose rates $\left(<1 \mathrm{~W} \cdot \mathrm{g}^{-1}\right)$. Moreover, a complete experimental parametrical study under laboratory conditions allowed the determination of the influence of the sensor geometry (horizontal fin, sensor height) for the same material structure (stainless steel), the influence of the material structure for a specific horizontal fin design, and the influence of coolant fluid conditions on the metrological characteristics of the prototypes (sensitivity, linearity, range) [7].

Thanks to these works, a new CALORRE configuration has been defined to target a nuclear absorbed dose rate up to $20 \mathrm{~W} \cdot \mathrm{g}^{-1}$. As the preliminary out-of-reactor calibration has never been realized for such a range, a new calibration system has been developed to apply up to $60 \mathrm{~W}$ (instead of 4.5 or $6 \mathrm{~W}$ used for previous differential calorimeters, cf. Fig.1). This paper will focus on this new configuration made of Aluminum, studied by coupling experimental works under laboratory conditions by the new calibration device, theoretical calculations with a 1D thermal model and 3D 
numerical simulations for predicting behavior under irradiated conditions.

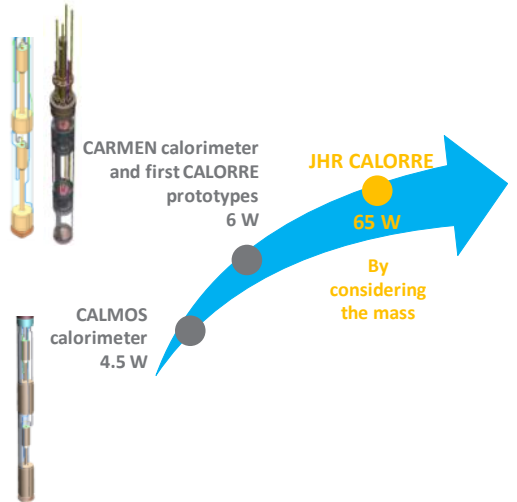

Fig. 1. Diagram of the evolution of the electrical power range applied to differential calorimeters for their calibration by simulating the nuclear absorbed dose rate by means of Joule effect.

The first part of the paper describes the experimental set-up including the new configuration of CALORRE, the new heating system composed of four independent 4-wire heaters used to imitate the nuclear absorbed dose rate by Joule's effect and the new operating protocol adapted for the novel heating system.

The second part is devoted to the experimental characterization of the response of the configuration by imposing an electrical power up to $60 \mathrm{~W}$ for different conditions. The independent heaters will be used to test the response of the sensor for four identical powers and for asymmetrical depositions. The response and the calibration curves are analyzed by applying a complete 1-direction theoretical thermal model taking into account conductive exchanges (those through the horizontal fin and those inside the gas layers located between the head and the vertical fin) and radiative exchanges between the outer surface of the head and the inner surface of the vertical fin. The contribution of each transfer component is given to explain the sensor sensitivity and the linearity of the calibration curve versus the electrical power.

In the last part, 3D thermal numerical simulations are shown to estimate and validate the whole differential-calorimeter response under irradiation conditions and compare it with a prediction based on a heat balance associated to calibration curves obtained under non-irradiation conditions.

\section{EXPERIMENTAL DEVICES}

\section{A. CALORRE Calorimeter}

The studied calorimeter CALORRE corresponds to a new heat-flow differential calorimeter (Fig.2 (a)) composed of two superimposed calorimetric cells allowing radial heat transfers (a cell with a sample and the second without to be used as reference cell and remove the nuclear absorbed dose rate due to the cell structure).

Each cell (a head surrounded by an horizontal fin and a vertical fin) is instrumented with two K-type thermocouples (close to the external surface of the sample holder and to the internal surface of the vertical fin respectively) and a heater to perform an out-of-pile calibration (cf. Fig.2 b,c). The studied calorimetric cell $(23.1 \mathrm{~mm}$ in height and $17 \mathrm{~mm}$ in external diameter) is made of Aluminum (AL5754) and owns a half horizontal fin with 8 metal sectors and 8 empty sectors.
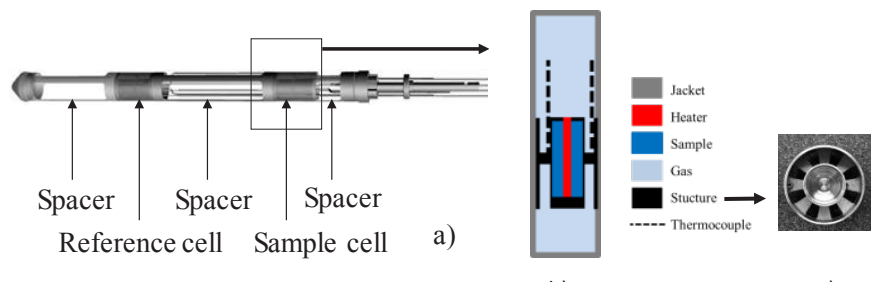

b)

Fig. 2. (a) Diagram of the differential calorimeter assembly composed of two superimposed cells, (b) Diagram of the calorimetric cell, (c) Photograph of the studied calorimetric cell.

The heater for this new configuration has been made especially to be able to inject up to $60 \mathrm{~W}$ during the out-of-pile calibration step, as said previously.

\section{B. Heating system}

The new home-made heating system is composed of four heating elements. Each heating element corresponds to a 4wire resistance consisting in alumina cylinder drilled with different vertical holes, $\mathrm{NiCr}$ wires, an Aluminum sample which holds the 4 heaters ( 4 connection wires per heater) (cf. Fig.3).

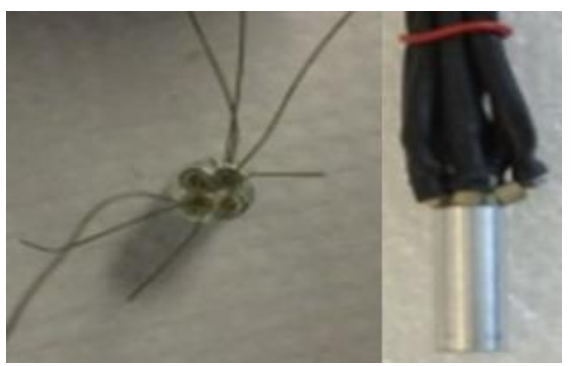

Fig. 3. Photographs of the heating system.

The nature of the material of the resistive wire was changed compared to previous employed heaters (Nickel-chromium instead of Constantan) to have a two times higher electrical resistivity (1.08 $\times 10^{-6} \Omega . \mathrm{m}$ against $5.2 \times 10^{-7} \Omega . \mathrm{m}$ respectively) leading to a greater resistance for the same length of resistive wire and thus a greater possible injected electrical power range (up to $15 \mathrm{~W}$ ).

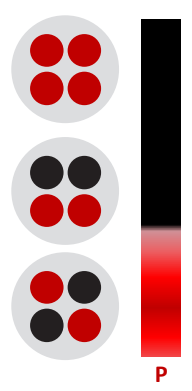

Fig. 4. Diagram for the different operating procedures applied to the heating system.

For the previous works, the power range was only from 0 to $4.5 \mathrm{~W}$ for CALMOS calorimeter [1] and up to $6 \mathrm{~W}$ for CALORRE or CARMEN calorimeters [2-7] (cf. Fig.1). 
The four heaters are assembled independently in order to apply different kinds of protocols: the same electrical power in each heater simultaneously, or different values in order to test the influence of a dissymmetric heating (cf. Fig.4).

\section{Experimental Set-Up}

The experimental set-up corresponds to the usual experimental set-up already used to realize the characterization of the temporal response of previous calorimetric cells under laboratory conditions and to do the out-of-pile calibration (cf. Fig.5) $[3-4,7]$. It is composed of a fluid circulating bath, 4 accurate resistive-shunts, 4 electrical power suppliers, 1 data acquisition device, 1 computer, $2 \mathrm{~K}$-Type thermocouples and the CALORRE assembly.

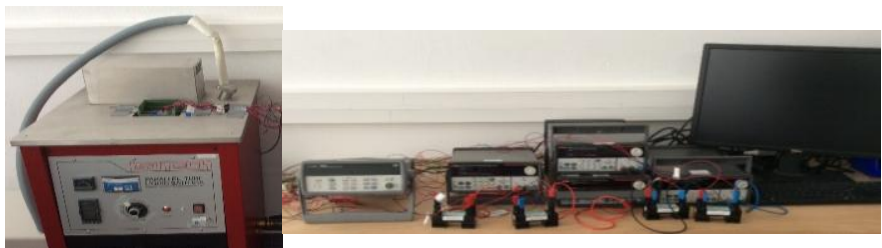

Fig.5. Photographs of the experimental set-up.

\section{RESPONSE UNDER LABORATORY CONDITIONS}

\section{A. Temporal response and calibration curves}

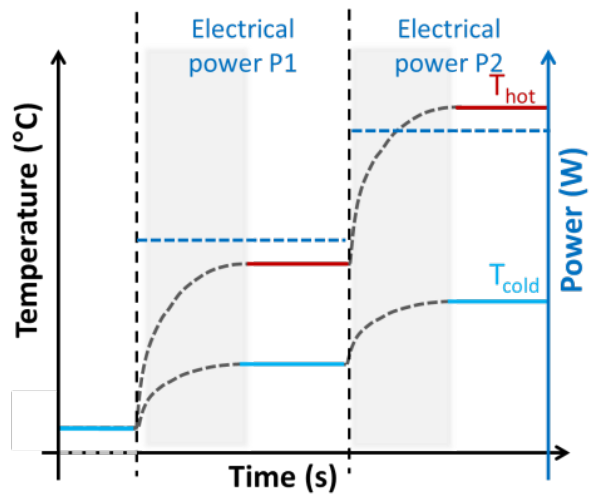

Fig. 6. Diagram of the operating procedure.

For each electrical power applied, the steady state is used to calculate the mean steady cold and hot temperatures $\left(T_{\text {hot }}\right.$ and $\left.\mathrm{T}_{\text {cold }}\right)$. Then the calibration curve is obtained by plotting the temperature difference $\left(T_{\text {hot }}-T_{\text {cold }}\right)$ versus the total electrical power applied to all heaters.

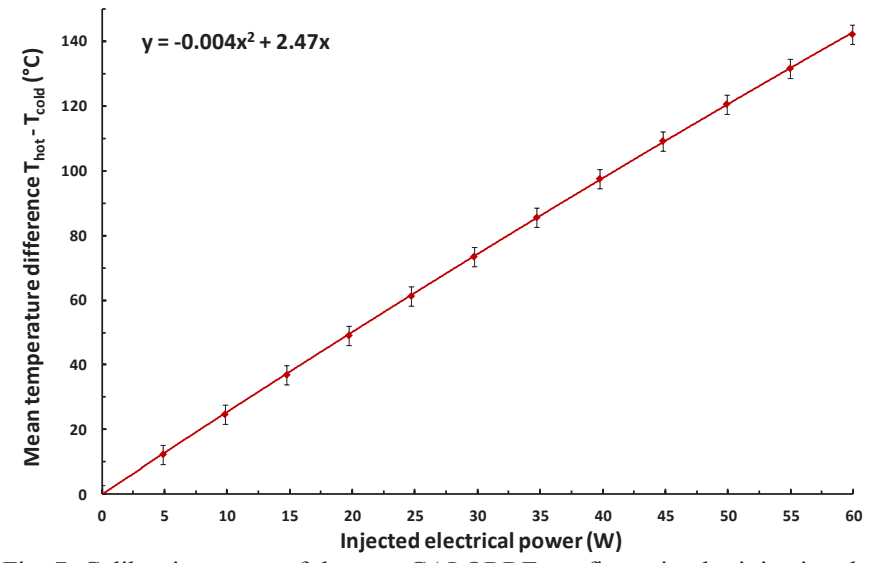

Fig. 7. Calibration curve of the new CALORRE configuration by injecting the same electrical power in each heating elements.
Figure 7 gives the calibration curve for the new configuration, it was obtained by averaging 4 calibration curves effectuated on 4 different days (reproducibility experiments).

\section{B. Result interpretation}

The calibration curve obtained for the new configuration is almost linear. Its sensitivity is equal to $2.47^{\circ} \mathrm{C} / \mathrm{W}$. Table 1 shows the results corresponding to other CALORRE configuration. This new CALORRE configuration is the less sensitive configuration but the most linear. By using Aluminum, its sensitivity is divided by 11.5 compared to the same structure geometry made on stainless steel.

TABLE I CALIBRATION FOR FOUR CALORRE CONFIGURATIONS (COOLING FLUID TEMPERATURE EQUAL TO $33^{\circ} \mathrm{C}$ )

\begin{tabular}{ccccc}
\hline \hline $\begin{array}{c}\text { Structure } \\
\text { material } \\
\text { nature }\end{array}$ & $\begin{array}{c}\text { Horizontal } \\
\text { fin } \\
\text { geometry }\end{array}$ & $\begin{array}{c}\text { Electrical } \\
\text { power } \\
\text { range } \\
(\mathrm{W})\end{array}$ & $\begin{array}{c}\text { Coefficient } \\
\mathrm{A} 1 \\
\left({ }^{\circ} \mathrm{C} . \mathrm{W}^{-1}\right)\end{array}$ & $\begin{array}{c}\text { Coefficient } \\
\mathrm{A} 2 \\
\left({ }^{\circ} \mathrm{C} . \mathrm{W}^{-2}\right)\end{array}$ \\
\hline Aluminum & Quarter fin & 6 & 5.04 & -0.018 \\
Aluminum & Half fin & 60 & 2.47 & -0.004 \\
Stainless steel & Quarter fin & 6 & 22.53 & -0.5 \\
Stainless steel & Half fin & 6 & 30.03 & -0.816
\end{tabular}

This is due to the higher value of the thermal conductivity of Aluminum compared to stainless steel $\left(\sim 130 \mathrm{~W} \cdot{ }^{\circ} \mathrm{K}^{-1} \cdot \mathrm{m}^{-1}\right.$ as against $\left.\sim 15 \mathrm{~W} .{ }^{\circ} \mathrm{K}^{-1} \cdot \mathrm{m}^{-1}\right)$.

By using a 1D thermal model including conductive and radiative transfers between the head, the horizontal fin and the vertical fin, the contribution of each transfer can be determined to interpret the behavior of the configurations [7].

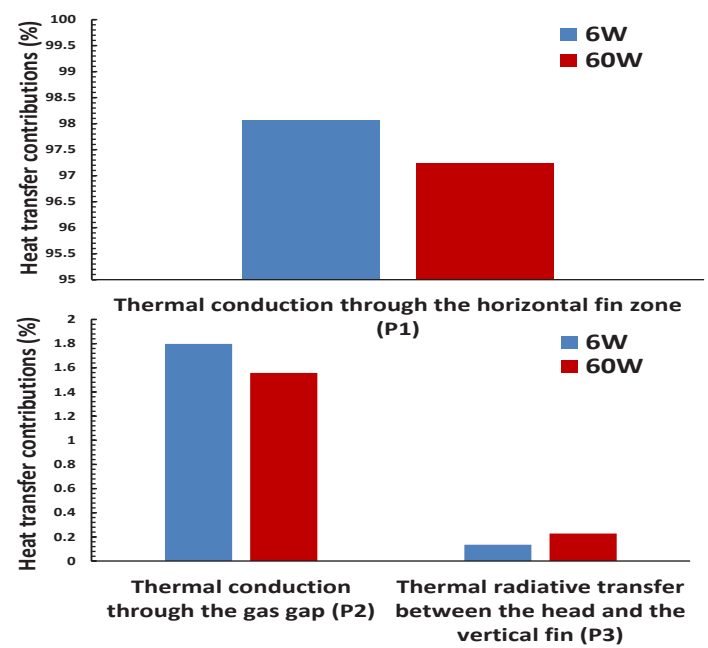

Fig. 8. Heat transfer contribution for the new CALORRE configuration for two applied electrical powers (6W in blue and $60 \mathrm{~W}$ in red): Thermal conduction through the horizontal fin in the upper figure, thermal conduction through the gas layer localized between the sample holder and the vertical fin and thermal radiative transfer between the sample holder and the vertical fin.

The predominant transfer is due to the thermal conduction through the horizontal fin (metal and gas sectors) (cf. Fig. 8). It corresponds to more than $97 \%$ of the total applied electrical power. The contribution of the thermal radiative transfer and that of the thermal conduction through the gas layer are very low $(<0.25 \%$ and $<1.6 \%$ at $60 \mathrm{~W}$ respectively). The thermal radiative transfer is very low because low absolute temperatures are reached $\left(<140^{\circ} \mathrm{C}\right.$ at $\left.60 \mathrm{~W}\right)$. Consequently, the associated thermal distribution leads to a liner response of the 
sensor on the wide power range (calibration curve with a negligible second-order coefficient).

\section{PREDICTION FOR REAL CONDITIONS}

The thermal behavior of the sensor under real conditions is simulated by using COMSOL Multiphysics 5.3a code. The heat equation is solved for the $3 \mathrm{D}$ domain corresponding to the differential calorimeter (two superimposed cells with spacers) and its jacket by neglecting wires (heaters and thermocouples). Thermal conductivities as a function of temperature are considered. Heat sources depending on the material nature are applied to all areas of the considered domain. Convective boundary limits are applied to the external surfaces of the calorimeter jacket by considering a heat transfer coefficient equal to $7325 \mathrm{~W} \cdot \mathrm{m}^{-2} \cdot \mathrm{K}^{-1}$. The cooling fluid temperature is equal to $33^{\circ} \mathrm{C}$.

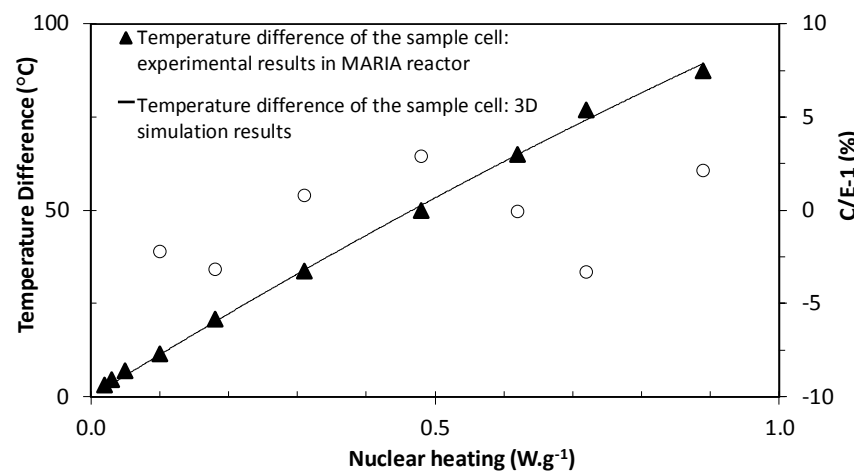

Fig. 9. Temperature differences obtained with the 3D simulations by using COMSOL Multiphysics 5.3a code and experimental temperature differences measured during the MARIA campaign (y-axis on the left) (H-IV-B channel, 24MWth, $\mathrm{h}=7325 \mathrm{~W} .{ }^{\circ} \mathrm{C}^{-1} \mathrm{~m}^{-2}$ and $\left.\mathrm{T}_{\mathrm{f}}=33^{\circ} \mathrm{C}\right)$. Relative deviation between numerical and experimental results (y-axis on the right).

Figure 9 gives results obtained with the configuration made of Stainless steel (non-linear response as seen in Table 1) inside the MARIA reactor (H-IV-B channel) at low nuclear heating $\left(<1 \mathrm{~W} \cdot \mathrm{g}^{-1}\right)$. A good agreement between the calculation and the experiment is observed (discrepancy from $-3.3 \%$ to $2.9 \%$ ) (remark: during this campaign, the distance between the two cells remained important because it was kept constant to be equal to the distance between the heads of the CARMEN cells (95mm) in order to do comparison).

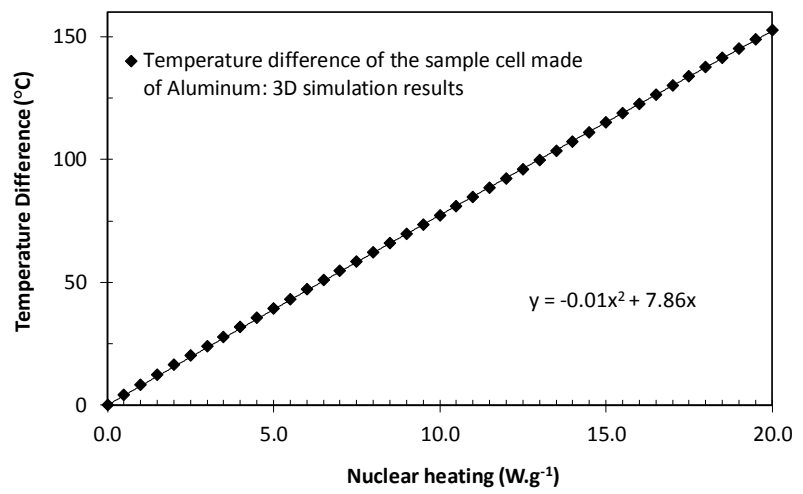

Fig. 10. Temperature differences of the sample cell of the differential calorimeter under irradiation conditions up to $20 \mathrm{~W} \cdot \mathrm{g}^{-1}\left(\mathrm{~h}=7325 \mathrm{~W} \cdot{ }^{\circ} \mathrm{C}^{-1} \mathrm{~m}^{-2}\right.$ and $\mathrm{T}_{\mathrm{f}}=33^{\circ} \mathrm{C}$ ).
Considering the validation of the 3D-model, the response of the new compact calorimetric sensor under real conditions and for high nuclear heating rates (up to $20 \mathrm{~W} . \mathrm{g}^{-1}$ ) can be estimated (cf. Fig. 10). The maximal temperature reached inside the calorimeter at $20 \mathrm{~W} \cdot \mathrm{g}^{-1}\left(258^{\circ} \mathrm{C}\right)$ is much lower than the melting point of Aluminum. This new configuration can be used for the highest nuclear heating rate that will be achieved in the core of the Jules Horowitz reactor at 100MWth by respecting safety margins.

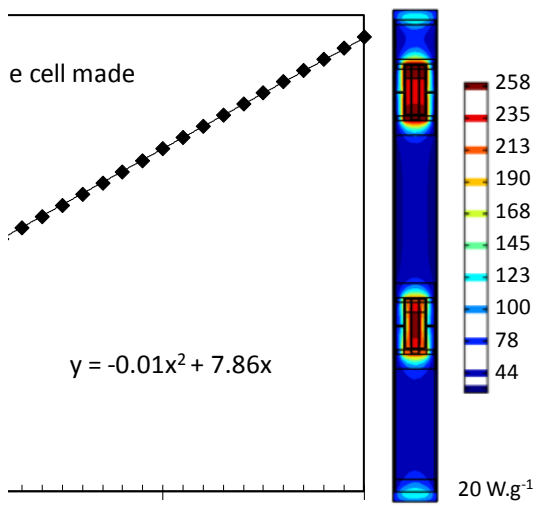

Fig. 11.Temperature field for the new CALORRE configuration for a nuclear heating rate equal to $20 \mathrm{~W} \cdot \mathrm{g}^{-1}\left(\mathrm{~h}=7325 \mathrm{~W} \cdot{ }^{\circ} \mathrm{C}^{-1} \mathrm{~m}^{-2}\right.$ and $\left.\mathrm{T}_{\mathrm{f}}=33^{\circ} \mathrm{C}\right)$.

\section{CONCLUSIONS}

Thanks to experimental, analytical and numerical works, a new configuration of the CALORRE differential calorimeter was characterized under laboratory conditions and for irradiation conditions (up to $20 \mathrm{~W} \cdot \mathrm{g}^{-1}$ ). A new heating system developed in order to calibrate the sensor for a great electrical power range which represents the total energy deposition in the calorimetric cell head was used successfully. Thanks to it, the power range is multiplied by 10 compared to the literature and previous calibrations (up to 60W). A linear calibration curve for this wide electrical power range was obtained. The linearity is explained by the $1 \mathrm{D}$ thermal model. The predominant heat transfer corresponds to thermal conduction through the horizontal fin (area where are localized the two Ktype thermocouples). The configuration was validated for high nuclear heating rates (up to $20 \mathrm{~W} . \mathrm{g}^{-1}$ ) thanks to $3 \mathrm{D}$ thermal simulations. Parametrical numerical studies will be carried out to optimize the calorimeter response and its size by reducing the inter-cell space in particular.

\section{REFERENCES}

[1] H.Carcreff, "CALMOS: Innovative Device for the Measurement of Nuclear Heating in Material Testing Reactors", Conference ISRD14, 2011.

[2] D. Fourmentel et al., "Nuclear Heating Measurements in Material Testing Reactor: A Comparison Between a Differential Calorimeter and a Gamma Thermometer", IEEE TNS, Vol. 60, Nº1, pp. 328-335, 2013.

[3] J. Brun et al., "Comparison of the thermal calibration response of two calorimetric cells dedicated to nuclear heating measurements", IEEE TNS, vol. 61, no. 4, pp. 2254-2261, Aug. 2014.

[4] J. Brun et al., "Responses of Single-Cell and Differential Calorimeters: from Out-of-Pile Calibration to Irradiation Campaigns", IEEE TNS, vol. 63, no. 3, pp. 1630-1639, June 2016.

[5] C. Reynard-Carette, et al., " Review Of Nuclear Heating Measurement By Calorimetry In France And USA", ANIMMA 2017 - Advancements in Nuclear Instrumentation Measurement Methods and their Applications, EPJ Web of Conferences 170, 04019 (2018). 
[6] J. Brun et al., "Irradiation Campaign in MARIA Reactor of an Innovative Calorimeter. Comparison of its Nuclear Heating Measurements with Two Kinds of Classical Sensors", ConferenceANIMMA), 2017.

[7] A. Volte et al., "Study of the Response of a New Compact Calorimetric Cell for Nuclear Heating Rate Measurements", IEEE Transactions on Nuclear Science, Vol. 65, N9, pp. 2461-2470, 2018. 